\title{
细菌几丁质酶基因的表达调控
}

谢池楚 ${ }^{1}$ ，贾海云 ${ }^{1}$ ，陈月华 1,2

1. 南开大学微生物学系, 天津 300071 ;

2. 分子微生物学与技术教育部重点实验室, 天津 300071

摘要: 几丁质酶可以降解几丁质, 广泛存在于各类微生物中。几丁质的降解产物几丁寊糖在医药、食品及农业 生防领域有很重要的应用价值及广泛的应用前景。细菌在利用几丁质时, 需要先分泌几丁质酶, 将几丁质降解 成几丁宑糖或单体, 再通过特异的转运系统送进细胞而被利用。胞内的几丁质降解产物作为特定的信号分子, 可以激活或阻遏相应 chi 基因的转录, 从而影响细菌几丁质酶的合成。在各种调节蛋白及应答元件的参与下, 细 菌几丁质酶的合成受到精密的控制。文章以链霉菌和大肠杆菌为代表综述了细菌在转运系统和基因表达两个层 面上控制几丁质酶合成的最新研究进展。

关键词：细菌；几丁质酶基因; 转运系统; 表达调控

\section{Regulation of chitinase genes expression in bacteria}

\author{
XIE Chi-Chu ${ }^{1}$, JIA Hai-Yun ${ }^{1}$, CHEN Yue-Hua ${ }^{1,2}$ \\ 1. Department of Microbiology, Nankai University, Tianjin 300071, China; \\ 2. Key Laboratory of Molecular Microbiology and Technology, Ministry of Education, Tianjin 300071, China
}

\begin{abstract}
Chitinases, which can hydrolyze chitin, occur in a wide range of microorganisms including viruses, bacteria, and fungi. The derivatives of chitin are potentially useful in several areas such as food processing, medicines, and biological control in agriculture. Some bacteria can uptake and utilize chitin as carbon source by secreting chitinase. The chitin is degraded into chito-oligosaccharides $\left[(\mathrm{GlcNAc})_{\mathrm{n}}\right]$ or $\mathrm{N}$-acetylglucosamine (GlcNAc) by chitinases, and then the chitin derivatives are transferred into cells by specific transport systems of bacteria. The intracellular chitin derivatives activate or suppress the transcription of a series of chi genes and affect the amount of chitinase. The expression of chitinase genes are strictly regulated by various regulatory factors and responsive cis-acting elements. The present review will focus on the transport system and the regulation of chitinase genes expression in bacteria.
\end{abstract}

Keywords: bacteria; chitinase gene; transport system; regulation

几丁质是 $N$-乙酰氨基葡萄糖(GlcNAc)的多聚物, 由几丁质酶降解后可以产生几丁单糖(GlcNAc)以及 几丁二糖 $\left[(\mathrm{GlcNAc})_{2}\right] 、 几 丁 三$ 糖 $\left[(\mathrm{GlcNAc})_{3}\right]$ 等分子
大小不同的几丁寡糖 $\left[(\mathrm{GlcNAc})_{\mathrm{n}}\right]$ 。几丁质是含量仅 次于纤维素的生物高分子物质, 广泛存在于自然界 中。如昆虫的甲壳及其中肠都存在大量的几丁质;

收稿日期: 2011-03-31; 修回日期: 2011-06-14

基金项目: 国家自然科学基金项目(编号 : 30971957)资助

作者简介: 谢池楚, 博士研究生, 研究方向：芽胞杆菌分子生物学。E-mail: xcc5275@mail.nankai.edu.cn

通讯作者: 陈月华, 教授, 研究方向: 芽胞杆菌分子生物学, 微生物遗传与育种。E-mail: yhchen@nankai.edu.cn

网络出版时间: 2011-8-22 10:05:01

URL: http://www.cnki.net/kcms/detail/11.1913.R.20110824.1111.001.html 
除卵菌外绝大多数真菌细胞壁都含有几丁质。因此 在农业应用方面, 几丁质酶可以加速害虫罹病进程, 抑制真菌生长, 起到防治农作物病虫害的作用。高 分子量的几丁寡糖有明显的抗肿瘤作用, 可激活人 巨噬细胞和淋巴细胞, 增强机体免疫力, 已广泛用于 抗癌的临床治疗以及药物及保健食品的开发领域 $[1,2]$ 。 最近, Nature报道了脂质几丁寡糖可作为信号分子 刺激植物内生真菌菌丝和菌根的形成, 进而刺激植 物根部发育 ${ }^{[3]}$ 。这是几丁寡糖在农业应用方面的最 新发现。

由于几丁质既可以作为微生物生长的碳源又可 以作为氮源, 因此在含有几丁质的土壤及海洋环境 中, 能够分解几丁质的微生物就具有一定的生长优 势。这些微生物也在进化过程中形成了严格控制几 丁质酶表达的机制。揭示细菌几丁质酶的调节机制, 可以明确几丁质酶合成过程中重要的调节因子及其 具体功能, 从而有目的地进行代谢工程遗传操作或 基因改造，更好地利用资源微生物的功能基因及其 蛋白。

对细菌几丁质酶合成控制的研究，国外从 90 年 代开始就有陆续系统的报道, 研究对象主要集中在 几类细菌 :如链霉菌(Streptomyces)、大肠杆菌(E. coli) 及几种海洋细菌等等。细菌在感知外界环境的几丁 质信号时, 首先需要相应的转运系统把作为能源物 质的信号转运进体内, 形成另一信号分子, 再诱导 细胞做出一系列复杂的反应。因此, 微生物是从转 运系统蛋白基因和几丁质酶编码基因两个水平上进 行调节, 控制细胞对几丁质的利用。本文也将从这 两个方面综述链霉菌等细菌的最新研究进展。

\section{1 细菌几丁质酶的诱导表达}

一般认为微生物几丁质酶基因受抑制物/诱导 物系统的调控, 葡萄糖及其容易利用的糖类明显抑 制酶的合成，而几丁单糖和分子大小不同的几丁寡 糖多为几丁质酶产生细菌的有效诱导物 ${ }^{[4]}$ 。目前报 道的许多细菌都遵循这一规律。例如, 在链霉菌中多 个几丁质酶基因的表达都受几丁质的诱导, 而在葡萄 糖存在的情况下几丁质酶的表达受到明显抑制 $[5]$ 。但 有些细菌并非如此简单。谢池楚等 ${ }^{[6]}$ 采用DNS法检 测 77 株苏云金芽胞杆菌(Bacillus thuringiensis, 简称 $\mathrm{Bt}$ )在有或无诱导物培养基中的几丁质酶活力, 发现
全部实验菌株在无几丁质诱导条件下，都能够保持 一定量的基础表达; 而添加诱导物之后, 许多 $\mathrm{Bt}$ 菌 株的几丁质酶表达兼具组成型和诱导型的特点, 表 型极为复杂, 表现出诱导合成的多态现象。

几丁质需要先降解成几丁寡糖或者单糖，进入 细胞内形成特定的信号分子才能诱导几丁质酶基因 的表达。不同细菌起诱导作用的物质可能不同，同 一菌株中不同的几丁质酶基因的诱导物也可能不一 致。链霉菌能够转运和利用几丁单糖和几丁二糖, 但是几丁单糖不能诱导链霉菌产生几丁质酶, 只有 转运进体内的几丁二糖才具有诱导作用 ${ }^{[7]}$ 。弧菌属 (Vibrio)中, 起诱导作用的物质是几丁二糖脱去一个 乙酰基形成的异壳二糖(GlcNAc-GlcN) ${ }^{[8]}$ 。粘质沙雷 氏菌(Serratia marcescens)几丁二糖酶的表达能够被 几丁单糖诱导, 但几丁二糖的诱导效率更高 ${ }^{[9]}$ 。

\section{2 细菌中几丁质水解产物转运系统}

\section{1 链霉菌和大肠杆菌}

细菌中, 很多糖类通过磷酸烯醇式丙酮酸 : 糖 类磷酸转移酶系统(Phosphoenolpyruvate:carbohydrate phosphotransferase system, PTS)进行转运。构成PTS 系统的 3 类蛋白中, 磷酸烯醇式丙酮酸 $(P E P)$ 依赖性 蛋白激酶 I (简称 E I ) 和耐热组氨酸-磷酸蛋白 (Heat-stable, histidine-phosphoryl protein, 简称HPr) 是非特异性的可溶性蛋白, 所有糖类PTS转运系统 都具有，而第三类是蛋白酶 II (简称 E II)，具有糖类 特异性。E II 一般包含 3 个结构域: 胞内可溶性蛋白 E II A和结合在细胞质膜上特异识别转运糖类的 $E$ II $\mathrm{B}$ 和 $\mathrm{E} \| \mathrm{C} \mathrm{C}^{[10]}$ 。

链霉菌和大肠杆菌都能通过PTS系统转运几丁 单糖。图 $1 \mathrm{~A}$ 显示了该转运系统中各种转运蛋白的位 置和功能。大肠杆菌几丁单糖PTS系统中的E II 由 $n a g-E B A C D$ 操纵子中的nag $E$ 编码 111$]$ 。而在链霉菌中 $\mathrm{E} \| \mathrm{B}$ 和 $\mathrm{E} \| \mathrm{C}$ 分别由基因 nagF和nagE2 编码 [12], E II $\mathrm{A}$ 由基因 $c r r$ 编码, $\mathrm{E} I \mathrm{~A}$ 参与细胞多种糖类的转运 [10]。几丁单糖通过PTS转运系统后形成 6-磷酸- $N$-乙 酰氨基葡萄糖(GlcNAc-6-P)，进一步受 6-磷酸- $N-$ 乙 酰氨基葡萄糖脱乙酰酶 NagA和 6-磷酸葡萄糖胺 (GlcN-6-P) 脱氨酶 NagB 作用降解成 6-磷酸果糖 (Fru-6-P)而被细胞利用 ${ }^{[13]}$ 。

不同细菌转运几丁二糖的系统不同。大肠杆菌 

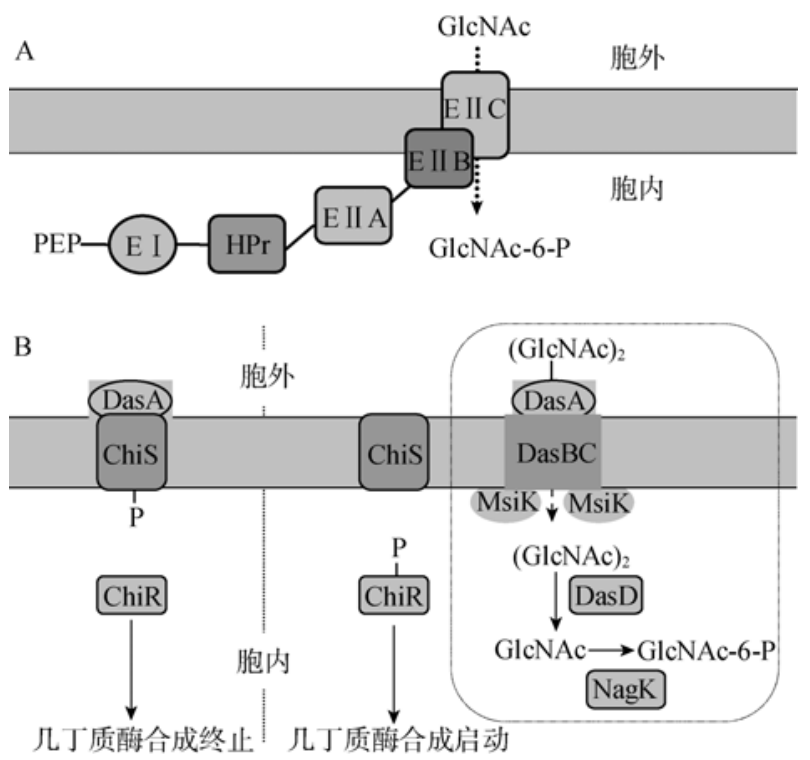

图 1 链䨓菌几丁质转运系统中各种转运蛋白的位置和 功能及双组份调节系统 ${ }^{[14]}$

A : 转运几丁单糖的 PTS 系统; B : 双组份调节系统。方框中为转运 几丁二糖的 $\mathrm{ABC}$ 转运子。

转运几丁二糖也是通过PTS系统。这一系统中的E II 由几丁二糖操纵子chb-BCARFG中的 $c h b B C A$ 三基因 编码。几丁二糖在转运过程中形成 6 位磷酸化的几 丁二糖 $\left[(\mathrm{GlcNAc})_{2}-\mathrm{P}\right]$, 该二糖由 $c h b F$ 编码的水解酶 降解为几丁单糖和 6-磷酸- $N$-乙酰氨基葡萄糖。降解 产生的几丁单糖可以分泌到胞外重新通过PTS系统 转运, 也可能被几丁单糖激酶作用, 两种途径都形 成 6-磷酸- $N$-乙酰氨基葡萄糖 ${ }^{[13]}$ 。

在链霉菌中, 几丁二糖由 ATP结合盒转运子 (ATP-binding cassette, 简称 $\mathrm{ABC}$ )负责转运。图 1B左 侧, ChiR处于抑制状态，细胞几丁质酶合成终止; 图 1B右侧, ChiR蛋白被激活, 酶的合成启动(详细解释 见后文)。图 1B右侧方框中显示 $\mathrm{ABC}$ 转运子全部参 与蛋白的位置及其功能。 $\mathrm{ABC}$ 转运子由胞外几丁二 糖结合蛋白 DasA和两种转运膜蛋白DasB和DasC构 成, 分别由 $d a s A B C$ 基因簇编码。 $\mathrm{ABC}$ 转运子在转运 几丁二糖的过程中还需要 $\mathrm{MsiK}$ 蛋白的参与, MsiK蛋 白与DasB和DasC相互作用行使功能 ${ }^{[7]}$ 。几丁二糖转 运进细胞内在 $N$-乙酰氨基葡萄糖苷酶(DasD)的作用 下降解成几丁单糖。而单糖的磷酸化是由几丁单糖 激酶 $(\mathrm{NagK})$ 完成。后续反应完全与几丁单糖的降解 途径相同 ${ }^{[14]}$ 。

不同链霉菌转运系统的功能也稍有差异。橄榄
绿链霉菌 (Streptomyces olivaceoviridis)ABC转运子 除能够转运几丁二糖外, 还能转运几丁单糖。天蓝 色链霉菌(S. coelicolor)中除 $\mathrm{ABC}$ 途径外，可能还有 另一转运几丁二糖的通道, 这种转运通道同样需要 MsiK蛋白的参与 ${ }^{[7]}$ 。

转运系统对于几丁质酶的诱导表达是必需的, 大肠杆菌转运蛋白编码基因 $c h b B$ 的突变型菌株就失 去诱导作用 ${ }^{[13]}$ 。天蓝色链霉菌 $m s i K$ 突变后, 几丁质 和几丁二糖对酶的诱导作用丧失 ${ }^{[7]}$ 。

\section{2 其他细菌}

许多研究证明, PTS转运系统和 $\mathrm{ABC}$ 转运子系 统也在其他革兰氏阳性菌和阴性菌中参与几丁单糖 和几丁二糖的转运和代谢。弗氏弧菌( V. furnissii)转 运几丁单糖的PTS系统与大肠杆菌类似, 由一系列 的nag基因编码的蛋白以及 $\mathrm{E}$ I、HPr蛋白组成, 此系 统对于几丁单糖的摄取和代谢非常重要 ${ }^{[15]}$ 。

粘质沙雷氏菌的PTS系统与大肠杆菌类似, 也 可以摄取几丁二糖，当破坏特异性 $\mathrm{E} \| \mathrm{C}$ 的编码基因 $c h b C$ 时, 几丁质酶活性相比野生菌株降低了 10 倍 [16]。导致动物患莱姆病的伯氏疏螺旋体 (Borrelia burgdorferi)也能通过PTS系统转运几丁二糖, E II C 的编码基因 $c h b C$ 对于几丁二糖的转运是必须的 ${ }^{[17]}$ 。

霍乱弧菌( V. cholerae)转运几丁二糖的是 $\mathrm{ABC}$ 转运子。10 个连续基因组成的基因簇可能构成了与 糖转运和利用相关的代谢操纵子。几丁质结合蛋白 (Chitin-binding protein, CBP)由其中的第一个基因编 码 118$]$ 。一种草酸杆菌Collimonas fungivorans含有与 链霉菌 $\mathrm{ABC}$ 转运子编码基因同源的基因, 可能也是 通过 $\mathrm{ABC}$ 转运子转运几丁二糖 ${ }^{[19]}$ 。

Yang等 ${ }^{[20]}$ 分析变形菌门(Proteobacteria)中一些 细菌的基因组, 比较了各菌株几丁单糖利用途径的共 同点和各自特性，证明交替单胞菌目(Alteromonadales) 和黄单胞菌目 (Xanthomonadales)的一些细菌中具有 新的转运几丁质相关的转运通道。Boulanger等 ${ }^{[21]}$ 证 实属于MFS(Major facilitator superfamily)类的转运 蛋白 $\mathrm{NagP}$ 是菜黄单胞杆菌(Xanthomonas campestris)转 运几丁单糖的主要通道, 这一通道是PTS系统和 $\mathrm{ABC}$ 型转运子以外的另一种转运单糖的系统, 该系 统在转运几丁单糖时不使其磷酸化。枯草芽胞杆菌 (B. subtilis) 在无葡萄糖时, 也是通过透性酶NagP转运 几丁单糖, 只是在转运过程中单糖被同时磷酸化 ${ }^{[22]}$ 。 


\section{3 细菌中几丁质水解产物转运系统的调控}

\section{1 大肠杆菌}

转运几丁单糖 PTS 系统中 E II 的编码基 因 $n a g E-B A C D$ 操纵子, 受其自身基因编码产物 $N a g C$ 和 $\mathrm{CAP}$ (Catabolite activator proteins)的调控。在这个操 纵子中, $n a g E$ 和其他几个基因的转录方向相反。但在 $n a g E$ 和 $n a g B A C D$ 的启动子区域都有 $\mathrm{NagC}$ 的结合位 点, 分别位于 $n a g E$ 基因转录起始位点上游-31 和-125 处, 这两个位点之间间隔 $94 \mathrm{bp}$ 。 $\mathrm{NagC}$ 在阻遏 $n a g E-B A C D$ 操纵子表达时, 结合在操纵位点上的两 个蛋白相互作用, 促使两位点之间的DNA形成环状 分子, 使相关基因无法转录。这一推论已通过体外 DNaseI足迹法和体内定点突变法分别被证实。 $\mathrm{cAMP} / \mathrm{CAP}$ 也参与阻遏复合物的组成, 但是它并非 抑制操纵子表达的必须阻遏蛋白, 可能是辅助阻遏 蛋白快速应答胞外的几丁单糖 ${ }^{[11]}$ 。

转运几丁二糖的PTS系统中编码E II 的操纵子 是 $c h b-B C A R F G$, 其中第一个基因 $c h b B$ 的 ORF位于 转录起始位点 $(+1)$ 下游 $107 \mathrm{bp}$ 处。该操纵子从 $+4 \sim$ $-112 \mathrm{bp}$ 为调控区域, 共有 5 个调节蛋白结合位点 (图 2), 与 5 个蛋白分子结合的DNA序列见图 2 各个 方框中序列。chb操纵子除受蛋白 $\mathrm{NagC}$ 和 CAP的调控 外, 还受其自身基因 $c h b R$ 编码产物 $\mathrm{ChbR}$ 的调节。 $\mathrm{NagC}$ 在 $c h b$ 操纵子结构基因调控区域有两个结合位 点, 上游结合位点以-112 处为中心, 下游结合位点 以+4 为中心, 并包含转录起始位点, 这两个结合位 点相距 $115 \mathrm{bp}$, 其中包含两个ChbR结合位点及位于 -83.5 处的一个与CAP结合的保守序列。ChbR1 和 ChbR2 可能以二聚体的形式结合在位于-38 和-61 的 一对含有 4 个不同碱基的 $19 \mathrm{bp}$ 正向重复序列上, 重 复序列之间间隔 $4 \mathrm{bp}$ 。抑制状态时, NagC和ChbR结 合在各自的结合位点上, 可能与 $\mathrm{cAMP} / \mathrm{CAP}$ 一起形 成一个复合物结构。当几丁二糖存在时进入诱导状
态, 诱导物是几丁二糖降解产生的 6 -磷酸- $N$-乙酰氨 基葡萄糖, 诱导 $\mathrm{NagC}$ 脱离两处结合位点而去阻遏。 此外, 在cAMP/CAP存在的条件下, 一个目前还未 知的ChbR诱导物 (可能是 6-磷酸几丁二糖) 使 ChbR 由阻遏物状态转变成激活蛋白状态, RNA聚合酶得 以结合在启动子上, 开始chb操纵子的转录 ${ }^{[13]}$ 。

\section{2 链霉菌}

对天蓝色链霉菌几丁单糖PTS转运系统的调控 研究得比较清楚。这一系统的激活蛋白为 $A \operatorname{trA}(T e t R$ 家族转录激活子), 而重要阻遏蛋白是全局性调节因 子DasR。E II B和 E II C 的编码基因 nagF和nagE2 均受 与抗生素基因表达相关的多效调节因子AtrA以及负 控制因子DasR的多重调节，两者结构基因上游都存 在DasR应答元件dre(DasR-responsive elements), 在 $n a g E 2$ 的启动子区域还存在 AtrA的特异结合序列 : GGAATCACGGGTTCC。dasR 基因突变的细胞, PTS 系统的转运过程由明显的诱导型变成组成型; 而 $\operatorname{atr} A$ 基因缺失的细胞, 其nagE2 的转录量明显降低, 转运蛋白减少。研究结果推导的诱导过程是：几丁 单糖通过PTS系统进入胞内降解成 6-磷酸葡萄糖胺, 该物质诱导阻遏蛋白 DasR变构, 进而从 $n a g F$ 和 $n a g E 2$ 启动子区域的结合位点脱离, 使 2 个基因去 阻遏而得以转录。同时, AtrA作为一种正调控蛋白激 活nagE2 的转录, 细胞便积累了转运蛋白 $\mathrm{E} / \mathrm{B}$ 和 $\mathrm{E}$ II $\mathrm{C}^{[12]}$ 。

序列分析证明, 在分别编码HPr、E I 和 II A 的 3 个基因 $p t s H 、 p t s I$ 和 $c r r$ 上游都有阻遏蛋白DasR的结 合序列。体外实验也证明，纯化的DasR能与这些基 因的启动子区域结合 ${ }^{[23]}$ 。因此, 变构的阻遏蛋白 DasR同样可以使这 3 个基因解除阻遏而得以转录,

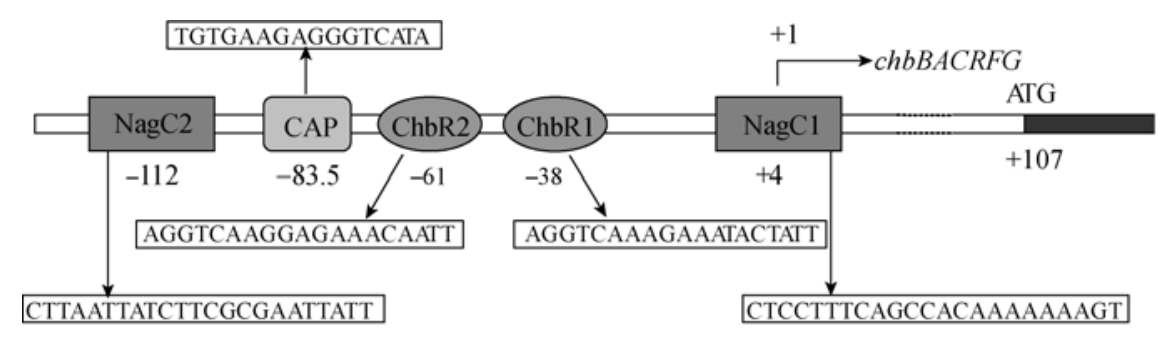


图 2 大肠杆菌几丁二糖转运系统编码基因 $c h b$ 操纵子上各个调节蛋白结合位点及其相应序列 ${ }^{[13]}$ 大量合成HPr、E I 和 II A，加速了PTS系统对几丁单 糖的转运 ${ }^{[24]}$ 。

在转运几丁二糖的 $\mathrm{ABC}$ 转运子亚基的编码基 因簇 $d a s A B C$ 中, d $a s A$ 单独转录, 而 $d a s B C$ 可能作为一 个转录单位同时转录。dasA也受DasR的调控。在das $A$ 结构基因上游 $106 \mathrm{bp}$ 处有一个 $d r e$ 位点。在 $d a s R$ 突变 的天蓝色链霉菌中, dasA的转录量提高了约两倍 ${ }^{[25]}$ 。

\section{3 其他细菌}

在变形菌门中, 除了大肠杆菌几丁质转运系统 受 $\mathrm{NagC}$ 蛋白调控外, 其他一些菌的转运通道可能受 $\mathrm{NagQ}$ 和NagR的调控 [20]。菜黄单胞杆菌中转运几丁 单糖的透性酶 $\mathrm{NagP}$ 就受 $\mathrm{NagQ}$ 的阻遏, nagQ基因突 变菌株 nagP的表达量明显增加 ${ }^{[21]}$ 。

Larsen等 ${ }^{[26]}$ 发现病原细菌单核细胞增生利斯特 氏菌(Listeria monocytogenes)的毒力基因调节因子 PrfA缺失, 几丁单糖和几丁二糖的摄取量减少, 几 丁质酶活力降低。在弗氏弧菌中, 缺失传感激酶 ChiS的突变细胞, 在含有几丁二糖的培养基中, 失 去几丁质趋化性和转运几丁质的特性。证明这些细 菌几丁质转运调节因子都是多功能的 ${ }^{[18]}$ 。

伯氏疏螺旋体几丁二糖PTS转运系统E II 的 3 个 编码基因 $c h b A 、 c h b B$ 和 $c h b C$ 中, $c h b C$ 的表达控制更 加严谨, 不仅受温度的影响 ${ }^{[27]}$, 该基因转录时还受 $\sigma$ 因子RpoD和RpoS的双重影响 ${ }^{[28]}$ 。

\section{4 细菌几丁质酶基因调控序列特征}

\section{1 链霉菌和大肠杆菌}

迄今为止, 对于链霉菌几丁质酶基因中与调节 蛋白结合的顺式作用元件(cis-acting elements), 即基 因调控序列，是研究和报道最为详尽的。早在 1992 年Delic等 ${ }^{[29]}$ 首次在 $P N A S$ 上报道, 链霉菌几丁质酶 基因上游有一对 $12 \mathrm{bp}$ 保守的正向重复序列 “TGGTCCAGACCT”，中间相隔 $17 \mathrm{bp}$ ，该序列与 预测的RNA聚合酶结合位点部分重叠, 并证明了这 段DNA参与了几丁质酶基因表达的调控。这是历史 上在链霉菌中第一个与几丁质利用有关的顺式作用 元件的发现。1997 年, PNAS上发表了同一实验室的 另一篇论文, 将几丁质酶基因chi63 上游中的正向重 复序列进行了定点突变，导致下游的报告基因组成
型表达，证明这一序列既与葡萄糖抑制又与几丁质 诱导有关, 认为该保守序列有可能是阻遏蛋白的结 合位点, 是一个负调控作用元件 ${ }^{[30]}$ 。

2007 年, Colson等来自 4 个不同国家的学者, 共 同完成了天蓝色链霉菌基因组中已克隆的 22 个不 同的几丁质代谢相关基因启动子序列的矩阵分析, 提出一段 $16 \mathrm{bp}$ 的共有序列是几丁质代谢相关基因 调节序列 ${ }^{[23]}$ 。全部 $c h i$ 基因都存在这一共有序列： “ANTGGTCTAGACCANT”，其中第 2 和第 15 个 碱基不确定。同时他们还发现，几丁单糖PTS转运系 统编码基因启动子序列也有一段几乎完全相同的 16 $\mathrm{bp}$ 的共有序列“ ACTGGTCTAGACCACT”，所不同 的是第 2 和第 15 个碱基确定为 $C$ 。并且经实验证明, 这 2 种保守的DNA序列是多效调节因子DasR的结合 位点, 作者将该保守序列称为DasR应答元件, 即 dre 位点, 这些DNA序列在体外都能与DasR结合。这一 $d r e$ 位点, 实际上与 1992 年发现的 $12 b p$ 的正向重复 序列极为相似。

大肠杆菌这方面的研究相对比较少。通过序列 分析, 发现在几丁质酶基因 $c h i A$ (也称为 $y h e B$ )结构 基因上游 $43 \mathrm{bp}$ 处，紧邻SD序列上游有一个CAP的 潜在结合位点, 其具体序列为：TTTGCAGTTTT TACGTCACAAGG。另外, 以 $-130 \mathrm{bp}$ 处为中心有一 个 $45 \mathrm{bp}$ 的回文序列 ${ }^{[31]}$ 。

\section{2 其他细菌}

Park等 ${ }^{[32]}$ 研究肠杆菌属G-1 菌株的基因 $\operatorname{chiA}$ 时 发现, 其启动子上游的一对反向重复序列(IRS)与 chiA基因在E. coli中的表达相关，不含IRS的启动子 片段比含有IRS的活性高 16 倍左右。而大肠杆菌自 身chiA基因并不含有类似的IRS。

本组研究工作中，发现地衣芽胞杆菌和苏云金 芽胞杆菌几丁质酶基因启动区域均含有 10 个碱基 左右的正向重复序列, 缺失这些序列的chi基因在大 肠杆菌细胞中，几丁质酶异源表达量明显提高，证 明该序列起到负控制元件的作用 ${ }^{[33]}$ 。这一正向重复 序列是否有调控芽胞杆菌自身 chi基因表达的功能正 在研究中。 


\section{5 细菌几丁质酶基因的表达调控}

\section{1 大肠杆菌}

Francetic等 ${ }^{[31]}$ 为了寻找大肠杆菌 $c h i A$ 基因表达 的调节基因, 采用 $\operatorname{Tn} 10$ 转座子随机插入基因组方法 发现, 多个chiA表达量提高的突变菌株都是由于全 局性调节蛋白H-NS编码基因hns受到破坏造成的。通 过竞争性凝胶阻滞分析, 调节蛋白H-NS与 $\operatorname{chi}$ 启动 子区域具有很高的亲和性。hns突变菌株表达ChiA的 量明显增多。同时一些影响H-NS 蛋白表达量的基 因如fis、crp 和 $s t p A$, 也不同程度地影响着 $c h i A$ 的表 达。stpA对 $h n s$ 的缺失有补尝作用, 两者都缺失的突 变细胞, 其 $c h i A$ 表达量比只有 $h n s$ 缺失的突变子要高, 但是仅 $\operatorname{stp} A$ 缺失突变则与野生菌株没有太大区别。 此外, 温度也影响 $c h i A$ 的表达, 当培养温度从 $37^{\circ} \mathrm{C}$ 降到 $30^{\circ} \mathrm{C}$ 时, chiA的表达量也增加。

\section{2 链霉菌}

链霉菌是许多抗生素的生产菌, 含有多种几丁 质酶, 而且有些已经用于工业, 因此对于链霉菌几 丁质酶基因表达调节方面的研究相对较系统和深 入。从目前研究报道来看, 链霉菌几丁质降解系统 的转录控制相当复杂, 远远不止起初大家认为相对 简单的操纵子一阻遏物的概念，而是具有相当多的 DNA 结合蛋白, 每一种蛋白都具有其自身的特异性 及特别的时间性。

链霉菌的几丁质酶基因受多种蛋白的调节。目 前已报道的调节因子有 5 种：双组份调节系统 ChiS 和 ChiR、多效调节因子 DasR、调节蛋白 Reg1、启 动子结合蛋白 $\mathrm{Cpb} 1$ 。下面分别介绍有关这些调节蛋 白最新研究结果。

\subsection{1 双组份调节系统}

1999 2000 年, 两组学者先后在热紫链霉菌 (S. thermoviolaceus $)^{[34]}$ 及天蓝色链霉菌 ${ }^{[35]}$ 中发现这些菌 株的几丁质酶编码基因上游的 chis和 chiR与菌株几 丁质酶基因的表达调节有关。chiS编码的蛋白 ChiS 结合在细胞膜上作为外界信号的感应器, 而 $c h i R$ 编 码的蛋白ChiR在细胞内与DNA结合, 调节目标基因 的转录。ChiS和ChiR构成的双组份系统, 调节着其 编码基因下游几丁质酶基因的表达。研究者发现, 在浅青紫链霉菌(S. lividans)中同时转入含有热紫链
霉菌 chis、chiR和chi40 3 个基因时, 菌株的几丁质 酶活力比仅转入chi40 单一基因时明显要高, 显然 2 种蛋白起到激活chi40 基因表达的作用。Colson等 ${ }^{[14]}$ 解释了它们的具体功能: 当细胞外只有几丁单糖时, 一种多效调节因子DasA结合在ChiS胞外的结构域 上, 使得 ChiS处于非激活状态, 此时整个系统处于 一种抑制状态(图 1B左侧); 当细胞外有几丁二糖的 情况下，几丁二糖与ChiS竞争结合DasA, Chis不与 DasA结合时则处于激活状态，使整个系统开启(图 1B右侧)。他们认为 Chis蛋白首先在其保守的组氨酸 位点(H 1199)自身磷酸化，之后磷酸基团转移到 ChiR保守的天冬氨酸位点(D 54), 使ChiR处于激活 状态，磷酸化的ChiR结合在chi基因的启动子上，便 激活了几丁质酶基因的表达。

然而，在天蓝色链霉菌中，基因 $c h i R$ 被破坏后， 基因 $c h i C$ 受几丁质诱导的效率明显下降 ${ }^{[36]}$, 但是 $c h i R$ 缺失菌株总的几丁质酶活力与野生菌株没明显 差异。可能双组份系统只影响紧邻其编码基因下游 几丁质酶基因 $c h i c$ 的表达, 其他几丁质酶基因不受 其调控, 照常表达, 这些基因表达的几丁质酶弥补 了ChiC降低所造成的影响 ${ }^{[35]}$ 。

\subsection{2 多效调节因子 DasR}

2002 年 $S e 0$ 等 ${ }^{[37]}$ 首次发现并证明DasR是一种 转录阻遏物。由于其编码基因 $d a s R$ 突变时导致灰色 链霉菌(S. griseus) 气生菌丝和孢子发育缺陷 (Deficient in aerial mycelium and spore formation), 故命名 该编码基因为 $d a s R$ 。在上述“转运系统的调控”节中已涉及到这一调节因子, 它不但调节转运系统 中的多种蛋白合成，也控制链霉菌中多数几丁质酶 基因的表达。随后, Rigali等 ${ }^{[38]}$ 通过计算机模拟分析 发现, 天蓝色链霉菌中 DasR与编码转运几丁单糖 PTS系统的多个基因以及自身编码基因有应答关系, 并通过体外实验证实DasR能与上述所有基因的启动 子区域结合。体外实验还显示 6-磷酸葡萄糖胺能够 抑制DasR与目标序列结合，作者据此认为 6-磷酸葡 萄糖胺是DasR行使阻遏功能的重要效应物 ${ }^{[24]}$ 。而 Saito等 ${ }^{[25]}$ 研究发现dasR的转录受几丁寡糖的诱导, 却不受几丁单糖的诱导。Colson等 [23]发现天蓝色链 霉菌多个几丁质酶基因的序列中都含有DasR蛋白应 答元件dre, 并通过实验证明这些保守序列在体外都 
能特异地与DasR结合。至于DasR具体如何影响几丁 质酶的表达, 还鲜有报道。在链霉菌中, 几丁单糖能 作为DasR的效应物却不能诱导几丁质酶的表达。因 此, 许多学者认为DasR是一个全局性调节因子, 与 几丁质酶基因的特异性转录因子相互作用而调节几 丁质酶基因的表达。

\subsection{3 调节蛋白 Reg1}

Reg1 蛋白与大肠杆菌LacI / GalR 调节蛋白家 族同源, 为调节基因(Regulatory gene)的缩写, 编码 345 个氨基酸, 其N-端具有DNA结合域。1997 年由 Nguyen等 ${ }^{[39]}$ 在浅青紫链霉菌中首次发现并报道。随 后他们通过对 Reg1 融合蛋白异源表达及体外DNA 结合实验证明, 该蛋白结合在被葡萄糖阻遏的多个 基因的启动子区域, 被称为多效调节蛋白。浅青紫 链霉菌的Reg 1 与枯草芽胞杆菌中的碳源分解代谢阻 遏物 CcpA具有 $31 \%$ 的相似性。在reg1 缺失的细胞中, 几丁质酶的诱导特性消失, 并且不受葡萄糖的阻遏, 成为组成型表达。体外DNA结合实验也显示Reg1 能 结合在菌株几丁质酶基因chi40 上游区域。这些结果 证明, Reg1 对于chi40 的诱导表达是必须的, 而它如 何影响葡萄糖的抑制作用目前还未知, 推测Reg1 可 能调节几丁质酶诱导物的转运系统 ${ }^{[40]}$ 。

\subsection{4 启动子结合蛋白 $\mathrm{Cpb} 1$}

几丁质酶基因启动子结合蛋白(Chitinase promoter binding, 简称 Cpb1)是 2005 年由Fujii等 ${ }^{[41]}$ 学者 发现。他们通过亲和纯化法, 从浅青紫链霉菌菌体 裂解液中, 纯化出一种特异性结合在其 $c h i A$ 基因启 动子区域上的蛋白, 并命名为 $\mathrm{Cpb} 1$ 。该蛋白中与 DNA结合相关的氨基酸序列与许多芽胞杆菌转录因 子的DNA结合域相似。在体外实验中, 大肠杆菌表 达的Cpb1 也能特异性结合 $c h i A$ 。他们发现, $c p b 1$ 突 变的细胞chiA诱导的效率与野生菌株接近, 但受葡 萄糖抑制的程度却明显降低。因此推测, Cpb1 很可 能是一个阻遏蛋白, 在碳源分解代谢抑制作用中直 接阻遏 $c h i A$ 基因的表达, 或者在此过程中作为一个 辅助因子协助或改变其他重要调节因子的功能。

另有学者报道, 葡萄糖激酶GlkA对链霉菌几丁 质酶基因的表达也有一定的调节作用 ${ }^{[42]}$ 。

\section{3 其他细菌}

\subsection{1 沙雷氏菌 $c h i$ 基因转录调节子 ChiR}

Suzuki等 ${ }^{[43]}$ 在研究粘质沙雷氏菌 2170 菌株几丁 质酶调控时, 发现调节基因 $c h i R$, 其编码蛋白的序 列与细菌中各种LysR型转录调节子有高度相似性。 当 $c h i R$ 发生突变时, 菌体不产生几丁质结合蛋白 CBP 21 和所有几丁质酶。说明ChiR是粘质沙雷氏菌 中几丁质利用基因的重要转录调节因子。

\subsection{2 弧菌及海洋细菌的调控因子}

杀鱼假交替单胞菌 (Pseudoalteromonas piscicida ) O-7 是一种海洋环境中有效降解几丁质的细 菌。Miyamoto等 ${ }^{[44]}$ 发现该菌株中存在 $\mathrm{CdsS} / \mathrm{CdsR}$ 双 组份信号传导系统, 该系统与前述链霉菌中 ChiS/ ChiR双组份系统类似。CdsR蛋白做为一个应答调节 因子, 被 $\mathrm{CdsS}$ 蛋白磷酸化后成为一个传感器激酶。 磷酸从 $\mathrm{CdsS}$ 到 $\mathrm{CdsR}$ 的转移作用是通过几丁质的降 级产物启动的。CdsR不仅结合在受几丁单糖诱导的 基因aprIV启动子区域，还结合在chiA、chiB、chiC、 $\operatorname{chiD}$ 四个几丁质酶基因和几丁质结合蛋白基因 $c b p 1$ 的启动子区域，这些基因全部受几丁单糖的诱导。 说明CdsR是该菌株中参与几丁质降解的全局性调控 因子。

$\mathrm{Li}$ 等 ${ }^{[18]}$ 发现另外 2 种海洋细菌弗氏弧菌和霍乱 弧菌具有 $A r c B$ 型的传感/激酶二元调控系统, 该系统 可以控制约 50 个基因的表达，其中许多与几丁质降 解有关。缺失传感激酶ChiS，几丁质酶的活性降低 且不受几丁二糖的诱导。

Defoirdt 等 ${ }^{[45]}$ 发现哈氏弧菌(V. harveyi)中几丁 质酶受群体感应的负调控, 群体感应系统完全失活 的突变菌, 其几丁质酶活力明显比该系统被完全激 活的菌株要高。

\subsection{3 病原性细菌的毒力基因中心调节因子}

病原细菌单核细胞增生利斯特氏菌, 降解几丁 质主要通过ChiA和ChiB两种几丁质酶。Larsen等 ${ }^{[26]}$ 发 现，该菌株致病毒力基因中心调节因子PrfA对几丁 质酶的表达具有重要的激活作用, $p r f$ 缺失突变子 相对于野生菌株几丁质酶活力明显降低。但PrfA如 何调控几丁质酶活性仍然不清楚, 因为在 $c h i A$ 和 chiB 启动子区域并没有发现结合PrfA 的特异DNA 
序列。

\section{6 展 望}

在几丁质酶基因表达调节的基础研究方面，链 霉菌和大肠杆菌研究得相对深入一些, 然而仍有些 细节需要进一步探索。由于转运系统蛋白位于细胞 膜上, 该系统在转运几丁质衍生物时, 影响衍生物 进入周质空间的蛋白也必然调节着几丁质衍生物的 转运 ${ }^{[46]}$, 其他影响因子及其作用有待进一步发掘。 另外，几丁质衍生物通过细胞壁的分子细节尚不明 确。尽管目前已经报道了链霉菌多种调节因子影响 着chi基因的表达，但这些调节因子的具体调节机制 还不是很清楚, 各调节因子之间是否有联系、如何 相互作用等一些问题仍无答案。随着功能基因组学 的深入研究, 随着蛋白与DNA、蛋白与RNA和蛋白 与蛋白之间相互作用研究技术的发展, 有望能在较 短时间内详细了解细菌几丁质酶合成控制机理及其 在整个代谢中所起的作用。我们就可以通过基因敲 除、基因改造、合成生物学等手段构建高量表达几 丁质酶的菌株或特殊用途菌株，使人类更好的利用 细菌几丁质酶。

其他细菌几丁质酶合成控制机理方面的研究工 作还刚刚起步。如革兰氏阳性的芽胞杆菌, 许多是 酶制剂的生产菌种，也不乏几丁质酶活性高的菌 株。苏云金芽胞杆菌是重要的细菌杀虫剂生产菌, 已经证明该菌株产生的几丁质酶对杀虫有明显地增 效作用 ${ }^{[47]}$, 地衣芽胞杆菌几丁质酶明显抑制真菌孢 子萌发, 国内外都有该菌株生产的杀菌剂 ${ }^{[48]}$ 。但这 些重要的芽胞杆菌chi基因表达调节方面的研究报道 几乎是零!

鉴于几丁质酶在生物防治、医药及保健方面的 广泛用途, 近 7 年来, 本实验室以生防芽胞杆菌为 材料，在几丁质酶基因表达类型、与表达调节相关 的启动子区域以及其他顺式作用元件等方面开展了 一些基础性探索工作，尚未涉及调节蛋白的研究。 国内也有一些实验室在从事其他细菌几丁质酶的基 础研究及开发。如何从已报道的链霉菌等微生物的 研究方法、研究结论中得到启发，去探讨芽胞杆菌 以及其他细菌几丁质酶基因表达的调节机理，最大 可能地利用这些资源细菌的功能蛋白，使其为人类
造福, 则是本篇综述的主要目的之一。

\section{参考文献(References):}

[1] Khoushab F, Yamabhai M. Chitin research revisited. Mar Drugs, 2010, 8(7): 1988-2012.DOI

[2] Zhao Y, Park RD, Muzzarelli RAA. Chitin deacetylases: properties and applications. Mar Drugs, 2010, 8(1): 24-46. $\underline{\mathrm{DOI}}$

[3] Maillet F, Poinsot V, André O, Puech-Pagès V, Haouy A, Gueunier M, Cromer L, Giraudet D, Formey D, Niebel A, Martinez EA, Driguez H, Bécard G, Dénarié J. Fungal lipochitooligosaccharide symbiotic signals in arbuscular mycorrhiza. Nature, 2011, 469(7328): 58-63. DOI

[4] Felse PA, Panda T. Regulation and cloning of microbial chitinase genes. Appl Microbiol Biotechnol, 1999, 51(2): 141-151. DOI

[5] Miyashita K, Fujii T, Saito A. Induction and repression of a Streptomyces lividans chitinase gene promoter in response to various carbon sources. Biosci Biotechnol Biochem, 2000, 64(1): 39-43. DOI

[6] 谢池楚, 陈月华, 蔡峻, 刘传, 陈艳玲. Bt几丁质酶的基 础表达及诱导合成的多态现象. 生物工程学报, 2010, 26(11): 1532-1538. DOI

[7] Saito A, Fujii T, Shinya T, Shibuya N, Ando A, Miyashita $\mathrm{K}$. The $m s i K$ gene, encoding the ATP-hydrolysing component of $N, N^{\prime}$-diacetylchitobiose ABC transporters, is essential for induction of chitinase production in Streptomyces coelicolor A3(2). Microbiol-Sgm, 2008, 154(Pt 11): 3358-3365. DOI

[8] Hirano T, Kadokura K, Ikegami T, Shigeta Y, Kumaki Y, Hakamata W, Oku T, Nishio T. Heterodisaccharide 4-O( $N$-acetyl- $\beta$-D-glucosaminyl)-D-glucosamine is a specific inducer of chitinolytic enzyme production in Vibrios harboring chitin oligosaccharide deacetylase genes. Glycobiology, 2009, 19(9): 1046-1053. DOI

[9] Toratani T, Shoji T, Ikehara T, Suzuki K, Watanabe T. The importance of chitobiase and $N$-acetylglucosamine (GlcNAc) uptake in $N, N^{\prime}$-diacetylchitobiose [(GlcNAC) $)_{2}$ ] utilization by Serratia marcescens 2170. Microbiology, 2008, 154(Pt 5): 1326-1332. DOI

[10] 马婉晴, 章珍, 刘悦琳, 王华忠. 大肠杆菌分解代谢产 物阻遏效应研究进展. 遗传, 2010, 32(6): 571-576. DOI

[11] Plumbridge J. Regulation of PTS gene expression by the homologous transcriptional regulators, Mlc and $\mathrm{NagC}$, in Escherichia coli (or how two similar repressors can behave differently). J Mol Microbiol Biotechnol, 2001, 3(3): 371-380. DOI 
[12] Nothaft H, Rigali S, Boomsma B, Swiatek M, McDowall KJ, van Wezel GP, Titgemeyer F. The permease gene nagE2 is the key to $N$-acetylglucosamine sensing and utilization in Streptomyces coelicolor and is subject to multi-level control. Mol Microbiol, 2010, 75(5): 11331144. DOI

[13] Plumbridge J, Pellegrini O. Expression of the chitobiose operon of Escherichia coli is regulated by three transcription factors: $\mathrm{NagC}, \mathrm{ChbR}$ and CAP. Mol Microbiol, 2004, 52(2): 437-449. DOI

[14] Colson S, van Wezel GP, Craig M, Noens EEE, Nothaft H, Mommaas AM, Titgemeyer F, Joris B, Rigali S. The chitobiose-binding protein, DasA, acts as a link between chitin utilization and morphogenesis in Streptomyces coelicolor. Microbiology, 2008, 154(Pt 2): 373-382. DOI

[15] Meibom KL, Li XB, Nielsen AT, Wu CY, Roseman S, Schoolnik GK. The Vibrio cholerae chitin utilization program. Proc Natl Acad Sci USA, 2004, 101(8): 25242529. DOI

[16] Uchiyama T, Kaneko R, Yamaguchi J, Inoue A, Yanagida T, Nikaidou N, Regue M, Watanabe T. Uptake of $N, N^{\prime}$-diacetylchitobiose [(GlcNAc $\left.)_{2}\right]$ via the phosphotransferase system is essential for chitinase production by Serratia marcescens 2170. J Bacteriol, 2003, 185(6): 1776-1782. DOI

[17] Nelson DR, Rhodes RG, Atoyan JA. The chitobiose transporter, $c h b C$, is required for chitin utilization in Borrelia burgdorferi. BMC Microbiol, 2010, 10:21. DOI

[18] Li XB, Roseman S. The chitinolytic cascade in Vibrios is regulated by chitin oligosaccharides and a two-component chitin catabolic sensor/kinase. Proc Natl Acad Sci USA, 2004, 101(2): 627-631. DOI

[19] Fritsche K, de Boer W, Gerards S, van den Berg M, van Veen JA, Leveau JHJ. Identification and characterization of genes underlying chitinolysis in Collimonas fungivorans Ter331. FEMS Microbiol Ecol, 2008, 66(1): 123-135. DOI

[20] Yang C, Rodionov DA, Li XQ, Laikova ON, Gelfand MS, Zagnitko OP, Romine MF, Obraztsova AY, Nealson KH, Osterman AL. Comparative genomics and experimental characterization of $\mathrm{N}$-acetylglucosamine utilization pathway of Shewanella oneidensis. J Biol Chem, 2006, 281(40): 29872-29885. DOI

[21] Boulanger A, Déjean G, Lautier M, Glories M, Zischek C, Arlat M, Lauber E. Identification and regulation of the $N$-acetylglucosamine utilization pathway of the plant pathogenic bacterium Xanthomonas campestris pv. campestris. J Bacteriol, 2010, 192(6): 1487-1497. DOI

[22] Resch M, Roth HM, Kottmair M, Sevvana M, Bertram R,
Titgemeyer F, Muller YA. Cloning, expression, purification, crystallization and preliminary $\mathrm{X}$-ray diffraction analysis of YvoA from Bacillus subtilis. Acta Crystallogr Sect F Struct Biol Cryst Commun, 2009, 65(Pt 4): 410-414. DOI

[23] Colson S, Stephan J, Hertrich T, Saito A, van Wezel GP, Titgemeyer F, Rigali S. Conserved cis-acting elements upstream of genes composing the chitinolytic system of streptomycetes are DasR-responsive elements. $J \mathrm{Mol}$ Microbiol Biotechnol, 2007, 12(1-2): 60-66. DOI

[24] Rigali S, Nothaft H, Noens EEE, Schlicht M, Colson S, Müller M, Joris B, Koerten HK, Hopwood DA, Titgemeyer F, van Wezel GP. The sugar phosphotransferase system of Streptomyces coelicolor is regulated by the GntR-family regulator DasR and links $N$-acetylglucosamine metabolism to the control of development. Mol Microbiol, 2006, 61(5): 1237-1251. DOI

[25] Saito A, Shinya T, Miyamoto K, Yokoyama T, Kaku H, Minami E, Shibuya N, Tsujibo H, Nagata Y, Ando A, Fujii $\mathrm{T}$, Miyashita K. The dasABC gene cluster, adjacent to dasR, encodes a novel $\mathrm{ABC}$ transporter for the uptake of $N$, $N$-diacetylchitobiose in Streptomyces coelicolor A3(2). Appl Environ Microbiol, 2007, 73(9): 3000-3008. DOI

[26] Larsen MH, Leisner JJ, Ingmer H. The chitinolytic activity of Listeria monocytogenes EGD is regulated by carbohydrates but also by the virulence regulator PrfA. Appl Environ Microbiol, 2010, 76(19): 6470-6476. DOI

[27] Tilly K, Elias AF, Errett J, Fischer E, Iyer R, Schwartz I, Bono JL, Rosa P. Genetics and regulation of chitobiose utilization in Borrelia burgdorferi. J Bacteriol, 2001, 183(19): 5544-5553. DOI

[28] Nelson DR, Rhodes RG, Coy W. Chitobiose utilization in Borrelia burgdorferi is dually regulated by RpoD and RpoS. BMC Microbiol, 2009, 9: 108.DOI

[29] Delic I, Robbins P, Westpheling J. Direct repeat sequences are implicated in the regulation of two Streptomyces chitinase promoters that are subject to carbon catabolite control. Proc Natl Acad Sci USA, 1992, 89(5): 1885-1889. $\underline{\mathrm{DOI}}$

[30] Ni XY, Westpheling J. Direct repeat sequences in the Streptomyces chitinase-63 promoter direct both glucose repression and chitin induction. Proc Natl Acad Sci USA, 1997, 94(24): 13116-13121. DOI

[31] Francetic O, Badaut C, Rimsky S, Pugsley AP. The ChiA (YheB) protein of Escherichia coli $\mathrm{K}-12$ is an endochitinase whose gene is negatively controlled by the nucleoidstructuring protein H-NS. Mol Microbiol, 2000, 35(6): 1506-1517. DOI

[32] Park JK, Okamoto T, Yamasaki Y, Tanaka K, Nakagawa T, 
Kawamukai M, Matsuda H. Molecular cloning, nucleotide sequencing, and regulation of the chiA gene encoding one of chitinases from Enterobacter sp. G-1. J Ferment Bioeng, 1997, 84(6): 493-501. DOI

[33] 肖亮. 地衣芽孢杆菌MY75 几丁质酶特性及表达调控元 件的研究[学位论文]. 南开大学, 2010. DOI

[34] Tsujibo H, Hatano N, Okamoto T, Endo H, Miyamoto K, Inamori Y. Synthesis of chitinase in Streptomyces thermoviolaceus is regulated by a two-component sensorregulator system. FEMS Microbiol Lett, 1999, 181(1): 83-90. DOI

[35] Kormanec J, Ševčíková B, Homérová D. Cloning of a two-component regulatory system probably involved in the regulation of chitinase in Streptomyces coelicolor A3(2). Folia Microbiol, 2000, 45(5): 397-406. DOI

[36] Homerová D, Knirschová R, Kormanec J. Response regulator ChiR regulates expression of chitinase gene, chiC, in Streptomyces coelicolor. Folia Microbiol, 2002, 47(5): 499-505. DOI

[37] Seo JW, Ohnishi Y, Hirata A, Horinouchi S. ATP-binding cassette transport system involved in regulation of morphological differentiation in response to glucose in Streptomyces griseus. J Bacteriol, 2002, 184(1): 91-103. DOI

[38] Rigali S, Schlicht M, Hoskisson P, Nothaft H, Merzbacher M, Joris B, Titgemeyer F. Extending the classification of bacterial transcription factors beyond the helix-turn-helix motif as an alternative approach to discover new cis/trans relationships. Nucleic Acids Res, 2004, 32(11): 3418-3426. $\underline{\mathrm{DOI}}$

[39] Nguyen J, Francou F, Virolle MJ, Guerineau M. Amylase and chitinase genes in Streptomyces lividans are regulated by reg1, a pleiotropic regulatory gene. J Bacteriol, 1997, 179(20): 6383-6390. DOI

[40] Nguyen J. The regulatory protein Reg1 of Streptomyces lividans binds the promoter region of several genes repressed by glucose. FEMS Microbiol Lett, 1999, 175(1): 51-58. DOI
[41] Fujii T, Miyashita K, Ohtomo R, Saito A. DNA-binding protein involved in the regulation of chitinase production in Streptomyces lividans. Biosci Biotechnol Biochem, 2005, 69(4): 790-799. DOI

[42] Saito A, Fujii T, Yoneyama T, Miyashita K. glkA is involved in glucose repression of chitinase production in Streptomyces lividans. J Bacteriol, 1998, 180(11): 29112914. DOI

[43] Suzuki K, Uchiyama T, Suzuki M, Nikaidou N, Regue M, Watanabe T. LysR-type transcriptional regulator ChiR is essential for production of all chitinases and a chitin-binding protein, CBP21, in Serratia marcescens 2170. Biosci Biotechnol Biochem, 2001, 65(2): 338-347. $\underline{\mathrm{DOI}}$

[44] Miyamoto K, Okunishi M, Nukui E, Tsuchiya T, Kobayashi $\mathrm{T}$, Imada $\mathrm{C}$, Tsujibo $\mathrm{H}$. The regulator $\mathrm{CdsS} /$ CdsR two-component system modulates expression of genes involved in chitin degradation of Pseudoalteromonas piscicida strain O-7. Arch Microbiol, 2007, 188(6): 619-628. DOI

[45] Defoirdt T, Ruwandeepika HAD, Karunasagar I, Boon N, Bossier P. Quorum sensing negatively regulates chitinase in Vibrio harveyi. Env Microbiol Rep, 2010, 2(1): 44-49. $\underline{\mathrm{DOI}}$

[46] Eisenbeis S, Lohmiller S, Valdebenito M, Leicht S, Braun V. NagA-dependent uptake of $N$-acetyl-glucosamine and $\mathrm{N}$-acetyl-chitin oligosaccharides across the outer membrane of Caulobacter crescentus. J Bacteriol, 2008, 190(15): 5230-5238. DOI

[47] Liu D, Cai J, Xie CC, Liu C, Chen YH. Purification and partial characterization of a $36-\mathrm{kDa}$ chitinase from Bacillus thuringiensis subsp. colmeri, and its biocontrol potential. Enzyme Microb Tech, 2010, 46(3-4): 252-256. $\underline{\mathrm{DOI}}$

[48] Xiao L, Xie CC, Cai J, Lin ZJ, Chen YH. Identification and characterization of a chitinase-produced Bacillus showing significant antifungal activity. Curr Microbiol, 2009, 58(5): 528-533. DOI

\section{•综合信息・}

\section{干细胞及其分化细胞彩色图谱}

郑月茂, 张翊华, 张雅蓉 著

978-7-03-031971-5 定价：88 元 装帧：平装 开本：16 分类：生物科学 
本图谱主要展示了编著者多年来科研积累的 252 幅干细胞及其分化细胞彩色图片, 同时展示了其他科研人员有关 胚胎干细胞、诱导型多能干细胞、造血干细胞和神经干细胞及其分化细胞的图片 6 幅。本书介绍了各类干细胞的特性、 分离培养和诱导分化。

欢迎各界人士邮购科学出版社各类图书

联系人 : 科学出版社科学销售中心 周文宇

电话 : 010-64017301 E-mail:zhouwenyu@mail.sciencep.com 